\title{
Does maternal diet during pregnancy influence clinical and laboratory characteristics of infantile-onset atopic dermatitis?
}

Chair and Department of Dermatology, School of Medicine in Katowice, Medical University of Silesia, Poland

\section{KEY WORDS}

Atopic dermatitis; diet; pregnancy; primary prevention; peripheral eosinophila; serum total IgE.

\section{Corresponding author}

Anna Lis-Święty

Chair and Department of Dermatology

School of Medicine in Katowice

Medical University of Silesia

Francuska 20/24

40-027 Katowice, Poland

E-mail: annadlis@neostrada.pl

Doi

10.23822/EurAnnACI.1764-1489.170

\begin{abstract}
Summary
Background. Prenatal environmental factors are suggested to be implicated in the dramatic increase in atopic dermatitis $(A D)$ in recent years. The aim of this study was to investigate the possible associations between pregnant woman's diet and clinical and laboratory variables of $A D$ in offsprings.

Methods. A cross-sectional study was performed in children 3-36 months of age with infantile-onset AD. Maternal dietary habits during pregnancy were evaluated in terms of the usual intake of dairy foods, eggs, red meat and poultry, fish, fruits and vegetables.

Results. One hundred pairs of mothers and their children with AD were included. A higher serum total IgE and peripheral eosinophila in children were associated with a lower maternal egg intake during pregnancy. Except for a strong trend toward significance of correlation between fish consumption and the lack of atopic multimorbidity, no relationships were revealed between clinical variables of child's $A D$ (the age of onset of $A D$, its severity, atopic multimorbidity) and the mother's dietary habit.

Conclusions. Our preliminary findings suggest that maternal egg intake during pregnancy might be a factor influencing laboratory markers of atopy in offsprings. Prospective cohort studies are needed to confirm and clarify this relationship.
\end{abstract}

\section{Introduction}

Atopic dermatitis (AD) is an important public health problem all over the world. This chronic, relapsing, intensely pruritic, inflammatory condition of the skin, in $60 \%$ of cases starts during the first 12 months of life and affects $20 \%$ and $35 \%$ infants and small children (1). Most children with infantile-onset AD develop other atopic diseases such as food allergies, asthma or allergic rhinitis later in life (2). Apart from genetics, environmental exposures, such as a pregnant woman's diet, are often considered to be possible factors in the development of $\mathrm{AD}$ and the socalled "atopic march" in children (2). Prescription of an antigen avoidance diet (including the exclusion of cow's milk, eggs, and peanuts) to a high-risk women during pregnancy, however, did not reduce atopic morbidity (3). A Western diet seems to have a causative effect, while the Mediterranean diet characterized by high intake of fruits, vegetables and fish is usually recommended as a healthy dietary pattern (4). Nonetheless, the link between prenatal dietary factors and $\mathrm{AD}$ is not well established.

The aim of this study was to examine the clinical and laboratory characteristics of the infantile-onset $\mathrm{AD}$ in relation to the maternal dietary habit during pregnancy.

\section{Materials and methods}

A cross-sectional study was carried out in mothers and their children with AD recruited consecutively at the Paediatric Dermatology Outpatient Clinic in Bielsko-Biała, Poland. Ethical approval from the Research and Ethics Committee of the Regional Medical Chamber in Bielsko-Biała and individual informed consent from each mother were obtained before the study was conducted. Participants had to meet the following inclusion criteria: children aged between 3 months and 36 months, $\mathrm{AD}$ diagnosed clinically according to the Hanifin and Rajka classi- 
fication (5), onset of $\mathrm{AD}$ symptoms within the first year of life, effective communication with mother. Other concomitant skin disorders in children, such as seborrheic dermatitis, psoriasis or ichthyosis were considered as exclusion criteria. Clinical data were obtained by face-to-face interviews and from the medical records. The severity of $\mathrm{AD}$ was evaluated according to the Rajka and Langeland grading (6), with the parameters of extent, course, and intensity. Depending on the score, the following disease subgroups were distinguished: mild (3.0-4.0), moderate (5.0-7.0) and severe $\mathrm{AD}$ (8.0-9.0). Coexistent allergic diseases like food allergy, asthma or allergic rhinitis were diagnosed by a physician. All children underwent blood investigations to determine their eosinophil count and serum total $\operatorname{IgE}(\mathrm{tIgE})$ levels. The number of eosinophils in peripheral blood samples was determined with the XS-1000i by automated blood haematology analyser 5 diff (Sysmex, Kobe, Japan). Serum tIgE level was measured using ELFA (Enzyme Linked Fluorescent Assay) technology (Vidas, bioMerieux SA, Lyon, France). All samples were tested in duplicate.

Data on maternal demographics (age at delivery, place of residence, educational level) and dietary habit during pregnancy were collected using a self-administrated standardized questionnaire, combining questions and statements already reported in the literature $(7,8)$. The following categories of foods were examined: dairy foods (milk, cheese and yogurt), eggs, red meat and poultry, fish, fruits and vegetables. The questionare concerned their usual intake with frequencies defined as: "never or less than once a month", "1-3 times a month", "once a week", "2-4 times a week", "5-6 times a week", "once a day", "2-3 times a day", "4-5 times a day" or " 6 or more times a day".

Statistical analysis of obtained data was performed with Excel and Statistica v.12 (StatSoft Inc., Tulsa, Oklahoma, USA). To create continuous variables and calculate descriptive statistics, categories of intake frequency for the different food items were converted to "times a day", "times a week" or "times a month" depending on the food item. Data were not found to be normally distributed, Kruskall-Wallis's test, U Mann-Whitney's tests and Kendall's rank correlation were therefore used when necessary to assess the relation of each food item on the AD clinical and laboratory variables. In all cases, $\mathrm{p}<0.05$ was defined as statistically significant.

\section{Results}

A total of 100 mother-child dyads participated in this study. The majority of them gave birth to a child at age between 20 and 29 years $(n=65)$, lived in urban areas with fewer than 100.000 people $(n=72)$ and the greater number of mothers had tertiary education $(n=76)$. Forty-seven of children were under 12 months old and 52 were males. AD began before the age of 3 months in 25 of the children, between the ages of 3 to 6 months in 38 , be- tween the ages of 6 to 9 months in 17, and between the ages of 9 to 12 months in 20 of the children. The median total objective AD severity score of the study subjects was 5.0 (IQR: 4.0-6.0). Forty-six of children had mild, 41 moderate and 13 severe AD. Atopic multimorbidity - coexistent allergic diseases like food allergy, asthma and/or allergic rhinitis (alone or associated) was present in 33 of the cases. Seventy-seven of children had a positive family history of atopy. Fifty-nine of children had raised peripheral eosinophil counts (>5\%), in 47 they ranged from 6 to $10.0 \%$, in 9 from 11 to $20 \%$, and in 3 they were greater than $20 \%$. In 83 of children tIgE levels were higher than $60 \mathrm{UI} /$ $\mathrm{mL}$, in 75 they ranged from 61 to $100 \mathrm{UI} / \mathrm{mL}$, in 4 from 101 to $1000 \mathrm{UI} / \mathrm{mL}$, in 6 from 1001 to $2000 \mathrm{UI} / \mathrm{mL}$, and in 2 they were greater than $2000 \mathrm{UI} / \mathrm{mL}$. Table I presents the associations between clinical and laboratory characteristics of $\mathrm{AD}$ and maternal dietary habit during pregnancy. An inverse relationship between child's serum $\operatorname{tgE}(\mathrm{t}=-0.24 ; \mathrm{p}<0.05)$, eosinophilia $(\mathrm{t}=-0.19 ; \mathrm{p}<0.05)$ and the maternal egg intake during pregnancy was the only statistically significant association. A higher maternal consumption of fish during pregnancy had a non-significant tendency towards a negative relationship with the appearance of atopic multimorbidity $(\mathrm{Z}=1.74 ; 0.081)$.

\section{Discussion}

Our study found no association between maternal dietary habit during pregnancy and the clinical variables of children with $\mathrm{AD}$, but the value of serum tIgE levels and blood eosinophil counts were lower in children if their mothers consumed more eggs during pregnancy. The correlation between severity of $\mathrm{AD}$ and the serum tgE as well as eosinophilia is well known (9). Infantile-onset $\mathrm{AD}$ seems to be associated with a higher total serum IgE level and high eosinophilia, suggesting that the disease possibly leads to more allergic sensitization and resulting in more severe eczema $(10,11)$. Baïz et al. (4) showed that preconceptional and gestational exposure of potential allergens, including egg allergens, may be beneficial for allergic rhinitis prevention in offsprings. In addition, they found that high consumption of eggs before pregnancy was inversely associated with the risk of wheezing, which may be an allergic disease symptom (4). There is also some evidence that a higher maternal intake of fruits, vegetables, fish, peanut, milk and wheat, during pregnancy may be associated with a lower risk of atopic diseases in children $(4,12)$. Conversely, a significant positive association was found between meat intake during the preconceptional period and a risk of $\mathrm{AD}$, wheezing and allergic rhinitis (4).

In the current research, except for a strong trend toward significance of correlation between fish consumption and the lack of atopic multimorbidity, none of the other food categories considered for analyses were linked with the clinical characteristics of $\mathrm{AD}$. The study has several important limitations. First, the 
Table I - Associations of clinical and laboratory characteristics of infantile-onset atopic dermatitis with the maternal dietary habit during pregnancy.

\begin{tabular}{|c|c|c|c|c|c|c|c|c|c|}
\hline & \multirow[t]{2}{*}{$\mathbf{H}$} & \multicolumn{2}{|c|}{$\begin{array}{c}\text { Age of atopic } \\
\text { dermatitis onset }\end{array}$} & \multicolumn{2}{|c|}{$\begin{array}{c}\text { Rajka \& Langeland } \\
\text { Score }\end{array}$} & \multicolumn{2}{|c|}{$\begin{array}{c}\text { Atopic } \\
\text { multimorbidity }\end{array}$} & \multirow{2}{*}{$\begin{array}{c}\text { Eosinophil } \\
\text { count }\end{array}$} & \multirow[t]{2}{*}{$\begin{array}{l}\text { Serum total } \\
\text { IgE level }\end{array}$} \\
\hline & & $\mathrm{P}^{*}$ & $\mathrm{H}$ & $\mathrm{P}^{* *}$ & Z & $\mathrm{P}^{* *}$ & Kendall's & & \\
\hline \multirow{5}{*}{ 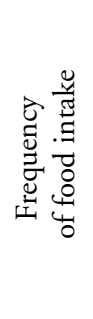 } & Dairy foods & 2,634 & 0,621 & 3,385 & 0,184 & 0,63 & 0,528 & $-0,01$ & $-0,12$ \\
\hline & Eggs & 6,930 & 0,140 & 1,766 & 0,414 & 1,19 & 0,233 & $-0,19$ & $-0,24$ \\
\hline & $\begin{array}{l}\text { Red meat } \\
\text { or poultry }\end{array}$ & 1,309 & 0,860 & 1,259 & 0,533 & 0,35 & 0,728 & 0,05 & 0,00 \\
\hline & Fish & 5,453 & 0,244 & 3,824 & 0,148 & 1,74 & 0,081 & 0,02 & $-0,06$ \\
\hline & $\begin{array}{l}\text { Fruits and } \\
\text { vegetables }\end{array}$ & 2,893 & 0,576 & 1,884 & 0,390 & 0,79 & 0,430 & $-0,10$ & $-0,10$ \\
\hline
\end{tabular}

${ }^{*} \mathrm{p}$ values obtained by Kruskal-Wallis test; ${ }^{* *} \mathrm{p}$ values obtained by Mann-Whitney $\mathrm{U}$ test; The values given in bold are $\mathrm{p}<0,05$.

use of self-administrated questionnaire to assess food intake can lead to errors as it may be subject to memory bias. The time since the delivery varied between participants, therefore some women had difficulty assessing their intake and more specific assessment of consumption data was not feasible. However, the aim of this research was to explore the overall dietary habit (frequency of intake of groups of foods) rather than details of dietary intake (portion sizes were not defined), which may have less of an impact on the availability of the information. To simplify the analysis, the questionnaire covered only important foods: dairy foods, eggs, red meat and poultry, fish, fruits and vegetables, and the results were related to these individual foods. Low correlation coefficients were found, so a clinically relevant connection could not be demonstrated. Moreover, this was a retrospective cross-sectional study and a control group was missing, thus causality could not be confirmed. Alternative mechanisms could be at play here.

Knowledge of factors determining disease severity in small children with $\mathrm{AD}$ is of great importance for preventive measures and optimizing care. Current findings indicate a balanced and diverse diet during pregnancy without restrictions (3). However, further studies in cohorts of pregnant women or infants are needed to match the proper strategies in this issue (3).

\section{Conclusions}

Our results suggest that maternal egg intake during pregnancy might be a factor influencing serum tIgE level and eosinophila in infantile-onset $\mathrm{AD}$. Additional research is warranted for a better understanding of this relationship.

\section{Conflict of interests}

The authors declare that they have no conflict of interests.

\section{References}

1. Kowalska-Olędzka E, Czarnecka M, Baran A. Epidemiology of atopic dermatitis in Europe. J Drug Assess 2019;8:126-128.

2. Wan J, Mitra N, Hoffstad OJ, Yan AC, Margolis DJ. Longitudinal atopic dermatitis control and persistence vary with timing of disease onset in children: A cohort study. J Am Acad Dermatol 2019;81:1292-1299.

3. Venter C, Greenhawt M, Meyer RW, et al. EAACI position paper on diet diversity in pregnancy, infancy and childhood: Novel concepts and implications for studies in allergy and asthma. Allergy 2020;75:497-523.

4. Bä̈z N, Just J, Chastang J, et al. Maternal diet before and during pregnancy and risk of asthma and allergic rhinitis in children. Allergy Asthma Clin Immunol 2019;15:40.

5. Hanifin JM, Rajka G. Diagnostic features of atopic dermatitis. Acta Derm Venereol 1980;60(suppl):44-47.

6. Rajka G, Langeland T. Grading of the severity of atopic dermatitis. Acta Derm Venereol Suppl (Stockh) 1989;144:13-14.

7. Bethlehem J. Applied survey methods: A statistical perspective. Hoboken, NJ: Wiley, 2009.

8. Willett W (Ed). Nutritional Epidemiology. Oxford: Oxford University Press, 2012:pp. 70-95.

9. Hu Y, Liu S, Liu P, Mu Z, Zhang J. Clinical relevance of eosinophils, basophils, serum total IgE level, allergen-specific $\mathrm{IgE}$, and clinical features in atopic dermatitis. J Clin Lab Anal 2020;18:e23214.

10. Holm JG, Agner T, Clausen ML, Thomsen SF. Determinants of disease severity among patients with atopic dermatitis: association with components of the atopic march. Arch Dermatol Res 2019;311:173-182.

11. Seo E, Yoon J, Jung S, Lee J, Lee BH, Yu J. Phenotypes of atopic dermatitis identified by cluster analysis in early childhood. J Dermatol 2019;46:117-123.

12. Bunyavanich S, Rifas-Shiman SL, Platts-Mills TA, et al. Peanut, milk, and wheat intake during pregnancy is associated with reduced allergy and asthma in children. J Allergy Clin Immunol 2014;133:1373-1382. 